\title{
ERRATUM
}

\section{Carbon disulfide induces mitochondria-mediated apoptosis in Sertoli-germ cells coculture}

\author{
Wei Wang ${ }^{1,2, *}$, Zhen Zhang ${ }^{1,3, *}$, Yinsheng Guo ${ }^{1}$, Yu Dong ${ }^{1}$, Xiaoyu Huang ${ }^{1}$, Yijun Zhou ${ }^{4}$ \\ \& Guoyuan Chen ${ }^{1}$
}

(C) The Korean Society of Toxicogenomics and Toxicoproteomics and Springer 2015

Erratum to: Mol Cell Toxicol (2015) 11:175-185

DOI 1.1007/s13273-015-0016-4

In Fig. 6E, Bax and Bcl-2 has been changed.

The online version of the original article can be found under doi: 1.1007/s13273-015-0016-4.

${ }^{1}$ Key Lab of Environment and Health, Department of Occupational and Environmental Health, School of Public Health, Tongji Medical College, Huazhong University of Science and Technology, 13 Hangkong Road, Wuhan 430030, PR China

${ }^{2}$ Department of Quality Control, Jiangxi Cancer Hospital, Nanchang 330029, People's Republic of China

${ }^{3}$ Pudong New Area Center for Disease Control and Prevention, 3039

Zhang Yang Road, Shanghai 200136, People's Republic of China ${ }^{4}$ Department of Environmental Health, School of Public Health,

Shanghai Jiaotong University, Shanghai 200025, PR China

*These authors contributed equally to this work.

Correspondence and requests for materials should be addressed to

G.Chen( $\varangle$ guoychen@163.com,wangeternal@126.com) 
(A)

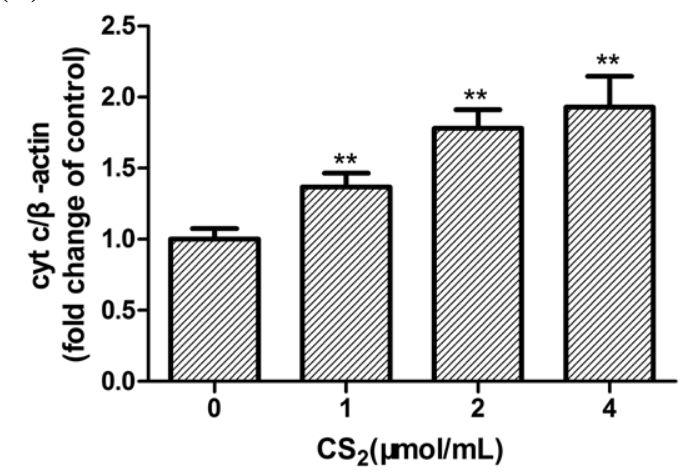

(C)

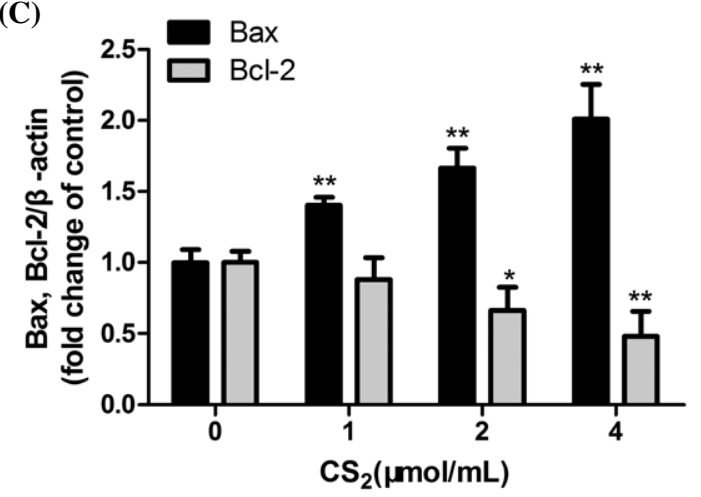

(E)
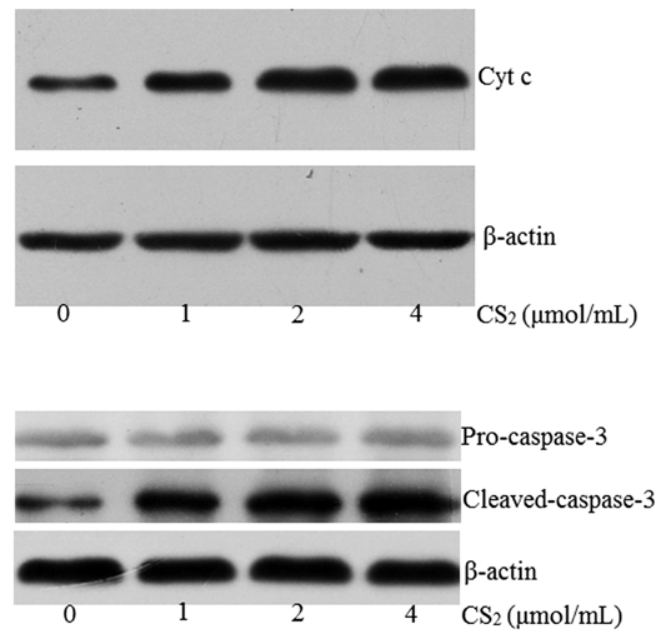

(B)

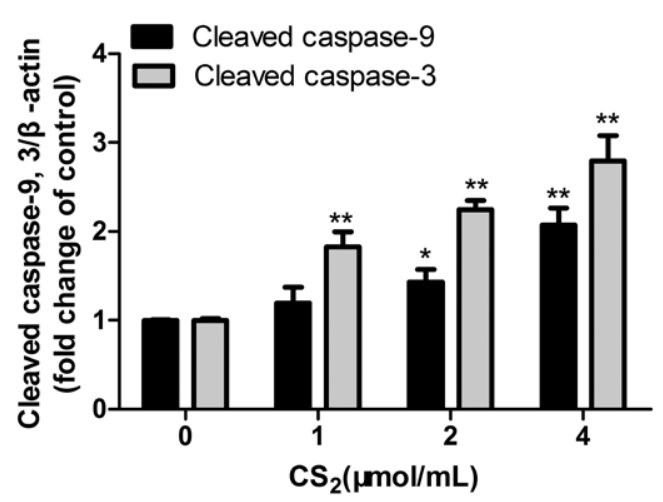

(D)
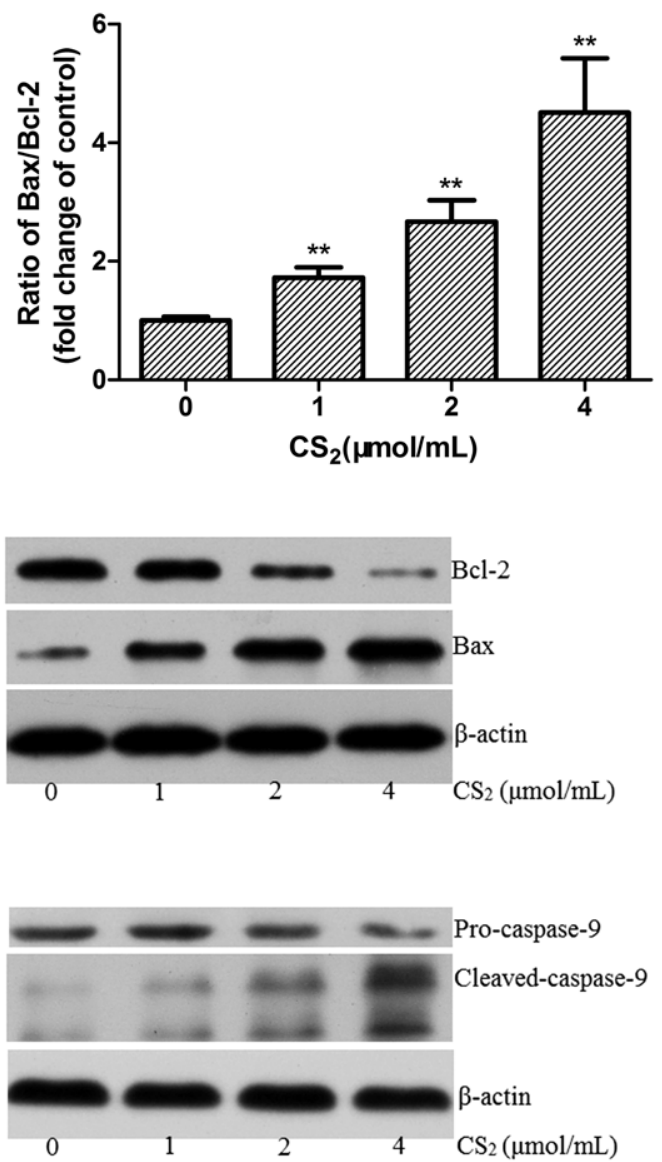

Figure 6. Effects of $\mathrm{CS}_{2}$ on protein levels of cytochrome c, the activation of caspases and Bcl-2 family members in Sertoli-germ cells. Sertoli-germ cells were treated with different concentrations of $\mathrm{CS}_{2}$ for $24 \mathrm{~h}$. (A) Relative levels of cytochrome c; (B) Relative levels of cleaved caspase-9 and caspase-3; (C) Relative levels of Bax and Bcl-2; (D) Relative levels of Bax/Bcl-2. (E) Total protein was extracted from treated Sertoli-germ cells and the protein levels of cytochrome c, Bax, Bcl-2, caspase-9 and caspase-3 were analyzed by western blot. $\beta$-actin was used as a loading control. Data are expressed as mean \pm SD. Compared with the control, $* P<0.05, * * P<0.01$. 